\title{
Risk factors associated with smoking behaviour in recreational venues: findings from the International Tobacco Control (ITC) China Survey
}

\author{
$\mathrm{X}$ Li, ${ }^{1,4} \mathrm{O} \mathrm{Li},{ }^{2,3} \mathrm{~L}$ Dong, ${ }^{4}$ B Sun, ${ }^{4}$ J Chen, ${ }^{4}$ Y Jiang, ${ }^{3}$ Y Yang, ${ }^{3}$ B Zhou, ${ }^{1}$ G T Fong ${ }^{2}$
}

\begin{abstract}
${ }^{1}$ Department of Epidemiology, School of Public Health, China Medical University, Shenyang, China; ${ }^{2}$ Department of Psychology, University of Waterloo, Waterloo, Ontario, Canada; ${ }^{3}$ National Center for Chronic and Non-communicable Disease Control and Prevention, Chinese Center for Disease Control and Prevention, Beijing, China; ${ }^{4}$ Shenyang Center for Disease Control and Prevention, Shenyang, China
\end{abstract}

Correspondence to:

Baosen Zhou, Department of Epidemiology, School of Public Health, China Medical University, No 92 Beier Road, Heping District, Shenyang, Liaoning Province, PR

China.110001; bszhou@

mail.cmu.edu.cn

Received 5 May 2009 Accepted 28 July 2009 Published Online First

10 August 2009

\begin{abstract}
Objective: To explore the determinants of smoking behaviour in recreational venues and to provide scientific bases for establishing smoke-free measures applying to these locations.
\end{abstract}

Methods: The International Tobacco Control (ITC) China Survey - a face-to-face cross-sectional survey of representative adult smokers from six cities (Shenyang, Beijing, Shanghai, Guangzhou, Changsha and Yinchuan) was conducted between April and August 2006. A total of 4815 smokers were selected using multistage sampling methods, and final analyses were conducted on 2875 smokers who reported patronising recreational venues at least once in the last six months. Multivariate logistic regression models were used to identify factors influencing the smoking behaviour within recreational settings. Outcome measure: Whether a smoker reported smoking in recreational venues during the last 6 months.

Results: $84 \%$ of subjects reported smoking in recreational venues. $32.0 \%$ of patrons reported partial or complete bans on smoking in these locations. The following factors were significant predicators of smoking in recreational venues: absence of bans on smoking, support for non-bans, being aged 18-24 years, positive smoking-related attitudes, low number of health effects reported and not living in Beijing.

Conclusions: The findings point to the importance of informing Chinese smokers about the active smoking and passive smoking harmfulness in both building support for smoke-free laws and in reducing smokers' desire to smoke within recreational venues. They also point to the importance of good enforcement of smoke-free laws when implemented. Such strategies could also serve to de-normalise smoking in China, a key strategy for reducing smoking in general.

Developing countries (with low and middle incomes) are facing a rapidly growing epidemic of tobacco use; rates in these regions began increasing in the early 1970s, and currently, $82 \%$ of the world's 1.1 billion smokers are in developing countries, with over $50 \%$ in Asia alone. ${ }^{1-3}$ One such country, the world's largest producer, consumer and victim of tobacco, is China. ${ }^{4}$ Currently, China is home to 350 million smokers $(30 \%$ of the world's smokers) and loses approximately one million people per year directly or indirectly because of tobacco-related deaths. ${ }^{5-7}$ If the smoking situation cannot be controlled effectively, it is estimated that about two million smoking-related deaths will occur among Chinese men by the year $2025,{ }^{8}$ and 200 million children currently living in
China will become smokers, 50 million of whom will die from smoking-attributable diseases. ${ }^{9}$ According to the Report on Tobacco Control in China for 2007, 540 million non-smokers are suffering from secondhand smoke, including 180 million children aged below $15 .{ }^{10}$

Recreational venues (for example, restaurants, coffee shops and karaoke lounges) that allow smoking expose people to contexts in which smoking may be viewed as the norm. This may encourage their progression to more regular smoking. ${ }^{11}{ }^{12}$ Further, the tobacco industry is actively promoting tobacco in recreational settings which may contribute to smoking uptake and relapse back to smoking for those trying to quit. ${ }^{13-15}$ Recreational venues tend to be frequented by the trend-setters in society: the elite, in China. If smoking goes on in these recreational venues, then people get the idea that smoking is acceptable and that smoking in these public places is just the way things are. Thus, smoke-free laws in recreational settings would be a powerful way to "de-normalise" smoking in China.

China's high prevalence of smoking and tremendous burden from tobacco-induced diseases make tobacco prevention an essential health priority. ${ }^{616}$ However, China currently has no smoke-free law at the national level, let alone one aimed at the population within recreational venues, which are all common venues for smoking and passive smoking exposure. Most current prevention programmes are based on the social influence approach, which targets the proximal psychosocial variables believed to promote individuals to smoke. ${ }^{17-19}$ Although such programmes are somewhat effective, the smoking-related risk factors utilised are based mainly on Western studies. ${ }^{20} 21$ Whether these factors have the same influence on Chinese smoking behaviour, especially in recreational venues, has not been identified to date. An examination of the behaviour, beliefs and characteristics of smokers who frequently patronise recreational venues may help in designing an appropriate and effective smoking prevention programme applying to these venues.

To bridge this data gap, the present study focused on smoking among adults within recreational settings. To the best of our knowledge, this is the first reported study to identify potential risk factors for smoking behaviour within recreational settings in China. In this study, we attempted to provide information on the determinants of smoking behaviour in recreational venues, and to develop a practical and effective 
smoking intervention strategy for recreational venues by examining the behaviour, beliefs and opinions of smokers who patronised these venues.

\section{METHODS}

This section provides an outline of the methods used in the ITC China Survey. A more detailed description can be found in the paper by Wu et al. ${ }^{22}$

\section{Sampling design}

This study was the baseline survey for the International Tobacco Control (ITC) China Survey, a cohort survey of adult smokers and non-smokers, designed to evaluate tobacco control policies. Survey waves are being conducted every year over a five-year period.

The ITC China Survey used a stratified multistage cluster sampling design in which six cities were first selected based on geographical representations and levels of economic development. These six cities were Shenyang, Beijing, Shanghai, Guangzhou, Changsha and Yinchuan. Within each city, 10 street districts (Jie Dao) were randomly selected, with probability of selection proportional to the population size of the Jie Dao. Within each of these Jie Dao, two residential blocks (Ju Wei Hui) were selected, again with probability of selection proportional to the population size of the Ju Wei Hui, from which a city-identified list of family households was used to sample 300 dwelling units (households) from every Ju Wei Hui using a simple random sampling method without replacement. Information on age, gender and smoking status for all adults living in these 300 households was collected. The enumerated 300 households were then randomly ordered, and individuals 18 years or older who had smoked at least 100 cigarettes in their lifetime were then approached following the randomised order until 40 adult smokers were surveyed. To increase the sample size for women smokers, one male smoker and one female smoker from every selected household were surveyed whenever possible. The next birthday method ${ }^{23}$ was used to select a respondent in households with more than one eligible male smoker.

\section{Procedure}

Once an individual was identified and agreed to participate, a face-to-face interview was scheduled. All interviews for adult smokers, lasting an average of 31 minutes, were conducted by trained research interviewers who administered a standardised questionnaire, including all the core items of the ITC policy surveys across the many countries (eight other countries at the time of the creation of the ITC China Survey) and some Chinaspecific measures. The same interview protocol was used across every city to ensure identical interview and data collection procedures. The present analysis is limited to respondents from Wave 1, conducted between April and August 2006.

\section{Measures}

\section{Demographic variables}

Information regarding present residential city, age, gender, ethnicity, education (no education or elementary school = "low"; junior high school or high school/technical high school = "medium"; college, university or higher = "high"), marital status, and per month household income (HH income) (where: <1000 yuan (1 yuan = £0.09; €0.1) = "low"; 10002999 yuan = "medium"; $\geqslant 3000$ yuan = "high"; don't know = "DK") was obtained through self-report. For daily smokers, we directly asked on average, how many cigarettes, including factory made and "hand-rolled" cigarettes, they smoke per day. Weekly smokers were asked for the average cigarettes they smoked per week.

\section{Reported smoking in entertainment venues}

Smokers were asked a series of questions on whether they had gone to each of several entertainment venues in the past 6 months, and for each venue, whether they had smoked. The entertainment venues were restaurants, coffee shops and karaoke lounges.

\section{Knowledge of health effects}

Knowledge of the harmful effects of smoking was assessed by asking the respondents if they believed that cigarette smoking can cause coronary heart disease (CHD), stroke, impotence, premature ageing, emphysema, stained teeth in smokers, lung cancer in smokers, lung cancer in non-smokers and addiction to tobacco.

\section{Extent of smoking restrictions}

Reported smoking restrictions for the recreational venues were assessed by asking: "Which of the following best describes the rules about smoking in indoor entertainment places such as restaurants, coffee shops, and karaoke lounges that you go most often?" Response options include: (1) smoking is not allowed in any indoor areas; (2) smoking is allowed only in some indoor areas; and (3) no rules or restrictions.

\section{Support for smoking restrictions}

Support for smoking restrictions for these venues were established by asking: "For the restaurants or bars venues, please tell me if you think smoking should be allowed in all indoor areas, some indoor areas, not allowed indoors at all or DK (don't know)?"

\section{Influence of surrounding friends/acquaintances}

Friends/acquaintances smoking behaviour's influence was identified by asking: "Of the five closest friends or acquaintances that you spend time with on a regular basis, how many of them are smokers?" Responses were rated on a six-point scale $(0=$ none, $1=$ one, $2=$ two, $3=$ three, $4=$ four and $5=$ five).

\section{Attitude and belief factors}

Smoking is a very common social practice in China and nonsmoking is not yet adopted as a social norm. The beliefs of smoking among smokers may influence their openness to smoking within recreational settings. These effects were measured with three statements. The first statement was "You enjoy smoking too much to give it up.", with a five-point scale from strongly disagree to strongly agree. In analysis, these who chose "strongly disagree" or "disagree" are identified as "disagree", on the contrary those who chose "strongly agree" or "agree" are identified as "agree". Then, all were asked "What do you think about the smoking behaviour?" The possible answers are very good, good, neither good nor bad, bad and very bad. Those respondents who chose "good" or "very good" are identified as having positive beliefs for smoking. The last statement was "What do you think about the attitude of Chinese society to smoking?", with a five-point scale: "support", "disapprove", "neither supports nor disapproves" or "DK, cannot say". 
Table 1 Demographic characteristics of those smoking in recreational venues vs those not smoking in those venues

\begin{tabular}{|c|c|c|c|}
\hline \multirow[b]{2}{*}{ Variables } & \multirow{2}{*}{$\begin{array}{l}\text { Smoking } \\
\text { No (\%) }\end{array}$} & \multirow{2}{*}{$\begin{array}{l}\text { Not smoking } \\
\text { No (\%) }\end{array}$} & \multirow[b]{2}{*}{ Test of significance } \\
\hline & & & \\
\hline Total & $2403(83.6)$ & $472(16.4)$ & \\
\hline \multicolumn{4}{|l|}{ Region } \\
\hline Beijing & $294(75.4)$ & $96(24.6)$ & \\
\hline Shenyang & $385(87.7)$ & $54(12.3)$ & \\
\hline Shanghai & $460(87.1)$ & $68(12.9)$ & \\
\hline Changsha & $387(83.4)$ & 77 (16.6) & \\
\hline Guangzhou & $484(84.3)$ & $90(15.7)$ & \\
\hline Yinchuan & 393 (81.9) & $87(18.1)$ & $\chi^{2}(5)=30.60, p<0.001$ \\
\hline \multicolumn{4}{|l|}{ Gender } \\
\hline Male & $2342(84.0)$ & $446(16.0)$ & \\
\hline Female & $61(70.1)$ & $26(29.9)$ & $\chi^{2}(1)=11.86, p=0.001$ \\
\hline \multicolumn{4}{|l|}{ Age } \\
\hline $18-24$ & $49(87.5)$ & $7(12.5)$ & \\
\hline $25-39$ & $515(83.5)$ & $102(16.5)$ & \\
\hline $40-54$ & $1290(87.3)$ & $187(12.7)$ & \\
\hline $55+$ & $549(75.7)$ & $176(24.3)$ & $\chi^{2}(3)=48.45, p<0.001$ \\
\hline \multicolumn{4}{|l|}{ Ethnicity } \\
\hline Han nationality & $2289(83.5)$ & $452(16.5)$ & \\
\hline Others & $114(85.1)$ & $20(14.9)$ & $\chi^{2}(1)=0.23, p=0.633$ \\
\hline \multicolumn{4}{|l|}{ Marital status } \\
\hline Single & $122(80.8)$ & $29(19.2)$ & \\
\hline Married & $2166(84.1)$ & $411(15.9)$ & \\
\hline Others & $115(78.2)$ & $32(21.8)$ & $\chi^{2}(2)=4.34, p=0.114$ \\
\hline \multicolumn{4}{|l|}{ Education } \\
\hline Low & $196(80.7)$ & 47 (19.3) & \\
\hline Medium & $1593(84.2)$ & $300(15.8)$ & \\
\hline High & $614(83.1)$ & $125(16.9)$ & $\chi^{2}(2)=2.10, p=0.351$ \\
\hline \multicolumn{4}{|c|}{$\begin{array}{l}\text { Household income per } \\
\text { month }\end{array}$} \\
\hline Low & $333(83.5)$ & $66(16.5)$ & \\
\hline Medium & $1566(83.7)$ & $306(16.3)$ & \\
\hline High & $326(83.8)$ & $63(16.2)$ & \\
\hline DK (don't know) & $178(75.7)$ & $57(24.3)$ & $\chi^{2}(3)=0.12, p=0.989$ \\
\hline \multicolumn{4}{|c|}{ Continuous variable } \\
\hline \multicolumn{4}{|l|}{ Age (years) } \\
\hline Mean (SD) & $47.36(8.53)$ & $50.41(11.86)$ & $\mathrm{t}(2873)=4.56, \mathrm{p}<0.001$ \\
\hline
\end{tabular}

\section{Data analysis}

The data were analysed using SPSS for Windows, version 13.0. Pearson's $\chi^{2}$ tests for categorical variables and $t$ tests for continuous variables were employed to examine differences between those smoking in recreational venues and those not. For further analyses of the association between selected factors and smoking behaviour in recreational venues, we conducted both bivariate and multiple logistic regression analyses. In the adjusted analyses, we added demographic variables along with the predictor variables to obtain adjusted odds ratios for each of the predictor variables and their corresponding $95 \%$ confidence intervals (AOR, 95\% CI). All analyses were conducted with weighted data using the "Complex samples" feature in SPSS to take the complex sampling design into account.

\section{RESULTS}

\section{Demographic characteristics of the sample}

The Wave 1 cooperation rates range approximately from $80.0 \%$ in Beijing and Guangzhou to $95.0 \%$ in Changsha. The response rates range from $39.4 \%$ in Yinchuan to $66.0 \%$ in Guangzhou. Data used in this study come from the 2875 smokers who completed the baseline survey and who reported visiting recreational venues during the last 6 months. The age of these respondents ranged between 18 and 82 years, with an average age of 47.9 (7.9) years.

Table 1 summarises the demographic characteristics of the subjects stratified by smoking status. A large majority (83.6\%) of the patrons reported ever smoking in these venues, with $84.0 \%$ for males and $70.1 \%$ for females, respectively. The majority of patrons were males $(97.0 \%)$, married $(89.6 \%)$ and of Han ethnicity (95.3\%). About $65.8 \%$ had completed high school education, and more than $86 \%$ reported per month household income greater than 1000 yuan. This is a high level of income relative to the average household income in China, which would be expected because the current sample was drawn from six major metropolitan cities in China, and because we restricted our analysis to those who went to recreational venues this population tends to have higher income.

\section{Bivariate results}

Comparisons of demographic characteristics between two groups are shown in table 1. Overall, both the educational attainment distribution and the marital status were almost equivalent across the two groups, and there were also no major differences regarding average monthly $\mathrm{HH}$ income ( $\mathrm{p}$ values not significant at the $\alpha$ level of 0.05 ). However, compared with 
Figure 1 Smoking behaviour within recreational settings among smokers who had been to these venues in the last six months across six urban cities of China.

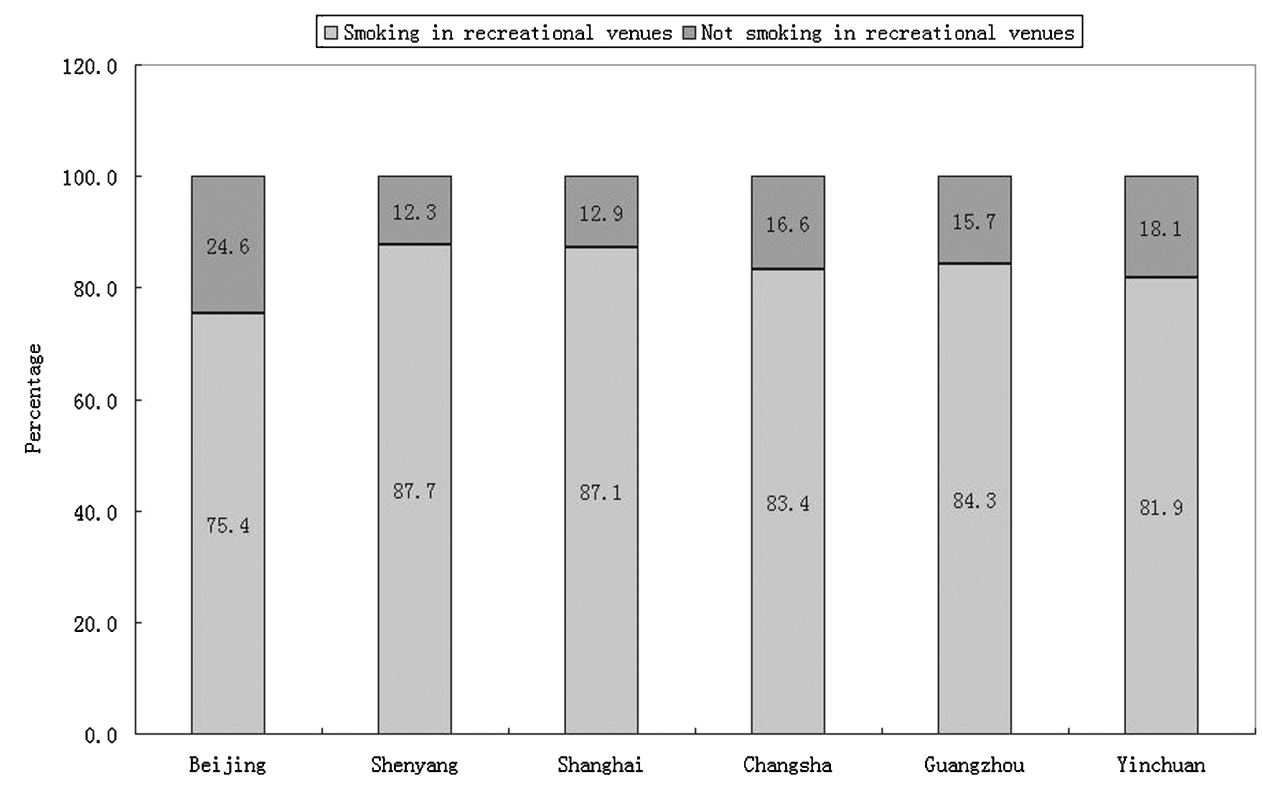

those not smoking in recreational venues, those smoking in recreational venues were more than three years younger (50.4 versus 47.4). Figure 1 presents the percentage of these smoking in recreational venues among all smokers reporting patronising recreational venues across six cities. A larger proportion of Shanghai and Shenyang respondents reported smoking in these venues compared to their counterparts in Changsha, Guangzhou and Yinchuan, with Beijing having the lowest percentage.

Associations between variables of interest and smoking behaviour within recreational venues are presented in table 2 . There was considerable variation $\left(\chi^{2}=386.1, p<0.001\right)$ in the reported extent of smoking restrictions. Only $32.0 \%$ of the patrons reported partial or complete bans on smoking in recreational venues of China, far fewer than that of bars in the United Kingdom, Canada, United States and Australia, which are considered to have the lowest levels of restrictions (fig 2). ${ }^{24-26}$ Those not smoking (55.5\%) in recreational venues were likely to express greater approval of bans on smoking, compared to those smoking (33.7\%). Great variation was also observed for all individual smoking-related health effects, with the exception of stroke $\left(\chi^{2}=3.55 ; p=0.06\right)$. Patrons were most likely to agree that smoking causes stained teeth $(87.7 \%)$ and lung cancer (72.9\%). However, less than half (49.0\%) agreed that smoking causes premature ageing. Only $36.2 \%$ and $18.1 \%$ agreed that smoking causes heart disease and impotence, respectively, and stroke was recognised by the lowest percentage of respondents as being caused by smoking (16.3\%) (fig 3). There was also a significant difference between groups in the total number of diseases endorsed by respondents $\left(\chi^{2}=27.6\right.$; $p<0.001$ ), with those smoking in recreational venues endorsing fewer diseases than those who did not smoke in recreational venues. Having a positive attitude towards smoking was more prevalent among those smoking in recreational venues. Those who perceived smoking as a good behaviour and that smoking is supported by Chinese society were more likely to smoke in recreational venues. "Enjoying smoking too much to give it up" was the most frequently cited reason for tobacco use within recreational settings. Those smoking in recreational venues were also far more likely to report having a greater number of their five closest friends who were also smokers.

\section{Multivariate results}

We next performed a complex sample multivariate logistic regression to assess the smoking risk factors within recreational settings. Table 3 displays the results of the logistic regression analyses, where the dependent variable was smoking vs not smoking in recreational settings. An overall opinion toward smoking of "very good" (AOR 30.64, 95\% CI 3.94 to 238.25) and reported no bans or restrictions on smoking (AOR 14.36, 95\% CI 9.41 to 21.91) were most strongly associated with smoking behaviour in recreational venues. Support for non-bans was also significantly related to this behaviour (OR 2.84, 95\% CI 2.09 to 3.86). The strength of this association was increased after adjustment for demographic characteristics (AOR 2.87, 95\% CI 2.12 to 3.89). Health knowledge of whether smoking causes lung cancer in smokers was associated with smoking behaviour within recreational settings (OR 0.63, 95\% CI 0.47 to 0.84 ), and this protective association was reduced (AOR 0.62, $95 \%$ CI 0.45 to 0.84 ) after adjusting the demographic characteristics including marital status, education attainment, ethnicity, and average monthly $\mathrm{HH}$ income, but remained significant. For all other smoking-related diseases mentioned, with the exception of stroke and stained teeth, similar findings emerged. Most notably, the odds of not smoking in recreational settings were greater among patrons who endorsed all the eight of health effects reported $\left(\chi_{\text {trend }}^{2}=28.4 ; \mathrm{p}<0.001\right)$. Those smokers living in Shenyang, Shanghai, Guangzhou, Changsha and Yinchuan were also more likely than those living in Beijing to smoke in recreational venues.

\section{DISCUSSION}

Bans on smoking in public spaces are becoming increasingly common in many countries. ${ }^{27-31}$ There are variations between countries where smoking is prohibited and in the strategies used to achieve these bans. In China, governments have imposed some smoke-free laws in public places, but many areas, especially recreational venues, such as restaurants and bars, have been exempted (fig 2). As a result, smokers within these settings have been able to smoke anywhere, at any time, and with little awareness of the dangers of smoking to themselves diseases, and increased in a linear fashion with the total number 
Table 2 Characteristics associated with whether smoking respondents reported smoking in recreational settings

\begin{tabular}{|c|c|c|c|}
\hline \multirow[b]{2}{*}{ Variables } & \multirow{2}{*}{$\begin{array}{l}\text { Smoking } \\
\text { No }(\%)\end{array}$} & \multirow{2}{*}{$\begin{array}{l}\text { Not smoking } \\
\text { No (\%) }\end{array}$} & \multirow[b]{2}{*}{ Test of significance } \\
\hline & & & \\
\hline \multicolumn{4}{|l|}{ Reported level of bans } \\
\hline Total indoor area & $124(5.2)$ & $131(27.8)$ & \\
\hline Some indoor area & $463(19.3)$ & $179(37.9)$ & \\
\hline No restrictions & $1816(75.6)$ & $162(34.3)$ & $\chi^{2}(2)=386.1, p<0.001$ \\
\hline \multicolumn{4}{|c|}{ Support for indoor restrictions } \\
\hline Total indoor area & $351(14.6)$ & $143(30.3)$ & \\
\hline Some indoor area & $459(19.1)$ & $119(25.2)$ & \\
\hline DK (don't know) & $113(4.7)$ & $33(7.0)$ & \\
\hline No restrictions & $1480(61.6)$ & $177(37.5)$ & $\chi^{2}(3)=107.6, p<0.001$ \\
\hline \multicolumn{4}{|c|}{$\begin{array}{l}\text { Smoking causes lung cancer in } \\
\text { smokers }\end{array}$} \\
\hline Yes & $1724(71.7)$ & $373(79.0)$ & \\
\hline No/DK & $679(28.3)$ & $99(21.0)$ & $\chi^{2}(1)=10.60, p=0.001$ \\
\hline \multicolumn{4}{|l|}{ Smoking causes CHD } \\
\hline Yes & $842(35.0)$ & $199(42.2)$ & \\
\hline No/DK & $1561(65.0)$ & $273(57.8)$ & $\chi^{2}(1)=8.66, p=0.003$ \\
\hline \multicolumn{4}{|l|}{ Smoking causes stroke } \\
\hline Yes & $379(15.8)$ & $91(19.3)$ & \\
\hline No/DK & $2024(84.2)$ & $381(80.7)$ & $\chi^{2}(1)=3.55, p=0.060$ \\
\hline \multicolumn{4}{|l|}{ Smoking causes impotence } \\
\hline Yes & 411 (17.1) & $110(23.3)$ & \\
\hline No/DK & $1992(82.9)$ & $362(76.7)$ & $\chi^{2}(1)=10.23, p=0.001$ \\
\hline \multicolumn{4}{|l|}{ Smoking causes emphysema } \\
\hline Yes & $1470(61.2)$ & $317(67.2)$ & \\
\hline No/DK & $933(38.8)$ & $155(32.8)$ & $\chi^{2}(1)=6.01, p=0.014$ \\
\hline \multicolumn{4}{|c|}{ Smoking causes stained teeth } \\
\hline Yes & $2102(87.5)$ & $420(89.0)$ & \\
\hline No/DK & $301(12.5)$ & $52(11.0)$ & $\chi^{2}(1)=0.83, p=0.361$ \\
\hline \multicolumn{4}{|c|}{ Smoking causes premature ageing } \\
\hline Yes & $1141(47.5)$ & $267(56.6)$ & \\
\hline No/DK & $1262(52.5)$ & $205(43.4)$ & $\chi^{2}(1)=13.03, p<0.001$ \\
\hline \multicolumn{4}{|c|}{$\begin{array}{l}\text { Smoking causes lung cancer in non- } \\
\text { smokers }\end{array}$} \\
\hline Yes & $1312(54.6)$ & $300(63.6)$ & \\
\hline No/DK & $1091(45.4)$ & $172(36.4)$ & $\chi^{2}(1)=12.86, p<0.001$ \\
\hline \multicolumn{4}{|c|}{$\begin{array}{l}\text { Total number of health effects } \\
\text { reported }\end{array}$} \\
\hline$\leqslant 1$ & $411(17.1)$ & $64(13.6)$ & \\
\hline 2 & $272(11.3)$ & $32(6.8)$ & \\
\hline 3 & $348(14.5)$ & $49(10.4)$ & \\
\hline 4 & $366(15.2)$ & $81(17.2)$ & \\
\hline 5 & $404(16.8)$ & $90(19.1)$ & \\
\hline 6 & $301(12.5)$ & $76(16.1)$ & \\
\hline 7 & $183(7.6)$ & $46(9.7)$ & \\
\hline 8 & $118(4.9)$ & $34(7.2)$ & $\chi^{2}(7)=27.6, p<0.001$ \\
\hline \multicolumn{4}{|l|}{ Tobacco is addictive } \\
\hline Disagree & $199(8.3)$ & $41(8.7)$ & \\
\hline Neither disagree nor agree & $133(5.5)$ & $37(7.8)$ & \\
\hline Agree & $2071(86.2)$ & $394(83.5)$ & $\chi^{2}(2)=3.95, p=0.139$ \\
\hline \multicolumn{4}{|c|}{$\begin{array}{l}\text { Enjoying smoking too much to give it } \\
\text { up }\end{array}$} \\
\hline Disagree & $823(34.2)$ & $193(40.9)$ & \\
\hline Neither disagree nor agree & $273(11.4)$ & $50(10.6)$ & \\
\hline Agree & $1307(54.4)$ & $229(48.5)$ & $\chi^{2}(2)=7.68, p=0.022$ \\
\hline \multicolumn{4}{|c|}{ Overall opinion of smoking behaviour } \\
\hline Very good & $42(1.7)$ & $1(0.2)$ & \\
\hline Good & $121(5.0)$ & $19(4.0)$ & \\
\hline Neither good nor bad & $1047(43.6)$ & $153(32.4)$ & \\
\hline Bad & $870(36.2)$ & $203(43.0)$ & \\
\hline Very bad & $322(13.4)$ & $96(20.3)$ & $\chi^{2}(4)=36.76, p<0.001$ \\
\hline
\end{tabular}


Table 2 Continued

\begin{tabular}{|c|c|c|c|}
\hline \multirow[b]{2}{*}{ Variables } & \multirow{2}{*}{$\begin{array}{l}\text { Smoking } \\
\text { No }(\%)\end{array}$} & \multirow{2}{*}{$\begin{array}{l}\text { Not smoking } \\
\text { No }(\%)\end{array}$} & \multirow[b]{2}{*}{ Test of significance } \\
\hline & & & \\
\hline \multicolumn{4}{|c|}{ Attitude of Chinese society to smoking } \\
\hline Disapprove/neither/DK & $1303(54.2)$ & $296(62.7)$ & \\
\hline Support & $1100(45.8)$ & $176(37.3)$ & $\chi^{2}(1)=11.51, p=0.001$ \\
\hline \multicolumn{4}{|l|}{ Cigarettes per day } \\
\hline 0-10 & $720(30.0)$ & $251(53.2)$ & \\
\hline $11-20$ & $1267(52.7)$ & $165(35.0)$ & \\
\hline $21-30$ & $212(8.8)$ & $29(6.1)$ & \\
\hline $31+$ & $204(8.5)$ & $27(5.7)$ & $\chi^{2}(3)=95.1, p<0.001$ \\
\hline \multicolumn{4}{|c|}{ Smokers of five closest friends } \\
\hline None & $37(1.5)$ & $15(3.2)$ & \\
\hline One & $66(2.7)$ & $25(5.3)$ & \\
\hline Two & $157(6.5)$ & $67(14.2)$ & \\
\hline Three & $402(16.7)$ & $91(19.3)$ & \\
\hline Four & $462(19.2)$ & $93(19.7)$ & \\
\hline All & $1278(53.2)$ & $181(38.3)$ & $\chi^{2}(5)=62.32, p<0.001$ \\
\hline
\end{tabular}

and others. The continuing problem of smoking in recreational venues has resulted in pressure on governments to ban smoking in these locations where it is still allowed. But the efficacious design of smoking control strategies for these venues is hampered by the relative absence of information, because few studies have focused specifically on this population. This study, to our knowledge, is the first publicly to explore the determinants of indoor smoking within recreational settings in China.

Tobacco consumers' beliefs about the harmfulness of secondhand smoke and their support for smoke-free laws are an important determinant of their smoking behaviour. For comparable respondents who supported no restrictions or bans in some indoor areas, the odds of smoking increased by $187 \%$ and $34 \%$, respectively (table 3 ). It has been well documented that an individual's own smoking behaviour influences their attitudes to tobacco control policies. ${ }^{32} 33$ However, these attitudes, in turn, may vary according to the enforcement status of restrictions. ${ }^{34}$ It could be argued that patrons in recreational venues without any smoking restrictions would be more susceptible to smoking. As shown in table 3, the odds of those who are exposed to limited bans or no bans at all to take up smoking more were 3.1 and 14.4 times higher than those exposed to total bans on smoking in recreational venues. Similar to previously published results, smokers were less likely to support smoke-free laws than non-smokers, ${ }^{35}$ with only $37.3 \%$ respondents showing support for such laws, a considerably lower rate than found in the ITC Four Country Survey. ${ }^{36}$ Additionally, our respondents who were in favour of smoking bans, more often advocated technical solutions (such as ventilation and smoking rooms) with support rates as high as $54 \%$; however, these concepts are often rejected by public health advocates.

The extent to which smokers understand the magnitude of these health risks has a strong influence on their smoking behaviour. Consistent with previous studies, ${ }^{37-39}$ both table 2 and table 3 indicated that smokers who haven't perceived greater health risk from smoking are more likely to smoke within recreational settings. Although there was a poor level of knowledge both in those smoking in recreational venues and those not (fig 3 ) in this study, the association increased after adjusting the demographic characteristics including marital status, education attainment, ethnicity and average monthly $\mathrm{HH}$ income (table 3). Different findings were noted by Smith ${ }^{40}$ and Rosliza, ${ }^{41}$ but Oncken ${ }^{42}$ and the present study showed evidence to support that the intention to give up smoking in public places is more prevalent among smokers with a good knowledge of the effects of smoking compared with smokers with poor knowledge. Figure 3 showed that only 18.1\% believed it could lead to impotence and only $17 \%$ acknowledged a potential relation between smoking and stroke risk. This illustrates the significant gaps in smokers' understanding of the risks of smoking. To bridge these gaps, antismoking education campaigns are needed in China.

The higher support rates in the US, Canada, UK and Australia are in large measure due to the dissemination of the strong evidence from many studies about the harmfulness of secondhand smoke. In these four countries, as in many other Western countries, knowledge about the harmfulness of secondhand smoke is considerably higher than in China. It thus would seem to follow that efforts to inform the Chinese public of the same studies about the harmfulness of secondhand smoke would help build the foundation for stronger support for smoke-free laws, and also for lowered prevalence of smoking in recreational venues and in other places where secondhand smoke would be particularly important to decrease or eliminate, such as in one's home and in cars, particularly in the presence of children.

The most important determinant of behaviour is behavioural intention, which, in turn, is influenced by one's overall evaluation of the behaviour (attitude).$^{43} \mathrm{~A}$ positive attitude towards smoking was more prevalent among those recreational venues smokers, as shown in table 2. The percentage of those smoking within recreational settings and those not, who enjoyed smoking too much to give it up, and who believed that Chinese society supported smoking, was $54.4 \%$ versus $48.5 \%$, and $45.8 \%$ versus $37.3 \%$, respectively. Parallel to the findings of previous research, ${ }^{44.46}$ positive beliefs about smoking were also found to be related to the smoking behaviour in public places. A smoker who responded with an attitude towards smoking of "very good" will be about 31 times more likely to smoke in public compared to a smoker who perceives it as "very bad".

Offering cigarettes to one another has become a means of social interaction and a friendly gesture, especially in entertainment spaces. Meanwhile, the need to gain social acceptance from peers exerting tacit pressure and influence also promotes smoking. To this end, smoking is used as a symbol of personality and independence. Therefore, it is no surprise to find that having "closest" smoking friends increased the odds of 
Figure 2 Reported prevalence of bans on smoking in recreational venues of China and bars of the UK, US, Canada and Australia, among smokers who had been to these venues in last six months.

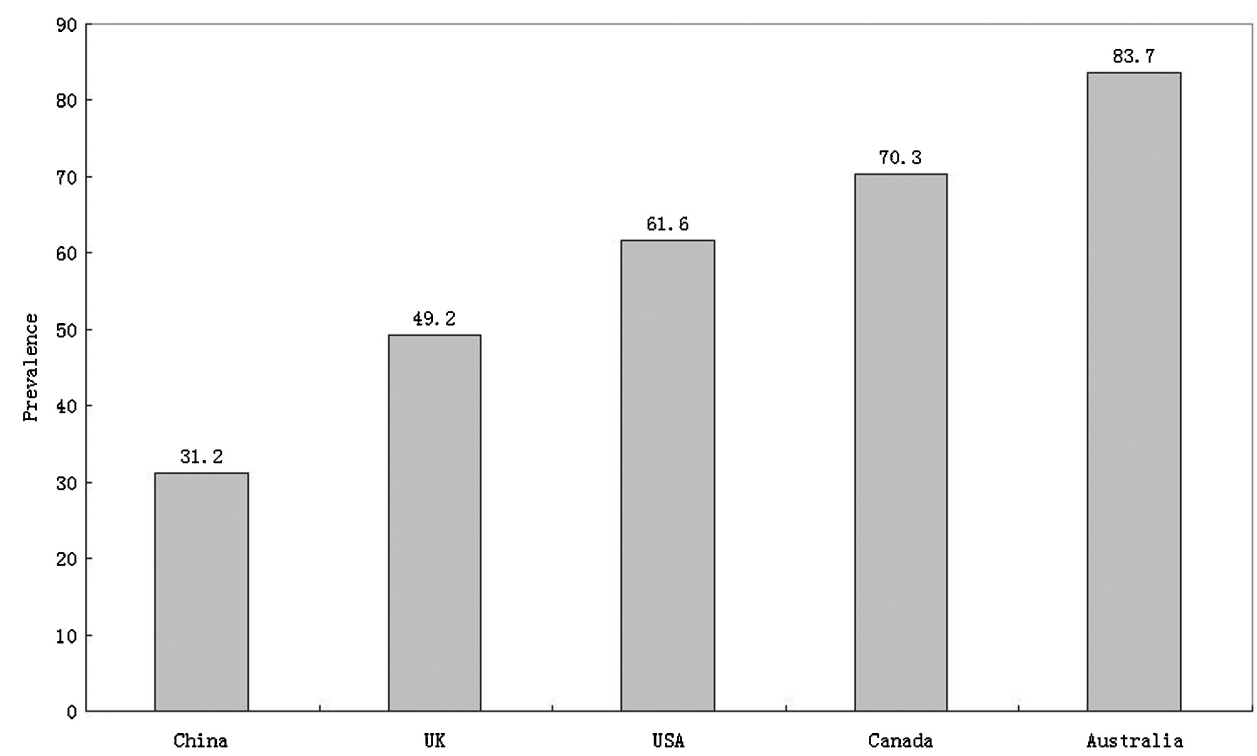

smoking more by 3.81 times, increasing in a linear fashion with the number of "closest" smoking peers reported (table 3 ). This was consistent with previously published reports. ${ }^{46-48}$ Of course, this phenomenon is possibly due to overstating the influence of peers, with selection and projection increasing the relation between peers' and smokers' behaviour. It could be argued that those who already smoke are more likely to seek out and spend time with other smokers, and those who smoke tend to overestimate the smoking prevalence of their friends.

As shown in table 3, compared to smokers in Beijing, smokers in Yinchuan, Changsha, Guangzhou, Shanghai and Shenyang are more likely to smoke in recreational venues, with the odds ratios ranging from 1.77 to 2.74 . This phenomenon may be partly due to the variations between districts where smoking is prohibited and in the strategies used to achieve these bans.

\section{Limitations}

Our results need to be considered in light of the following limitations. First, these results are based on the baseline data from the ITC China survey. The cross-sectional nature of these data cannot address the causality of the associations between variables. The second limitation of this study is the lack of information on the absent smokers. It is possible that absentees had a higher smoking possibility within recreational settings and a lower level of health effects than those surveyed. This may have caused underestimation of the smoking prevalence and the influence of the health effects. Third, cigarette smoking among women was traditionally unacceptable in Chinese culture. For a long time, this had served as a protective factor against smoking among women; so the sample and results of the present study are almost exclusively male. Thus, the suggested interventions based on the current study should be targeted more towards men. Finally, it is important to note that these results derive from smokers in the most affluent and most highly educated cities, with the most comprehensive tobacco control policies, in China. As such, the findings may not be generalised to the rest of the people living in rural areas. Similarly, we should expect health knowledge to be substantially lower among the majority of Chinese smokers,
Figure 3 "Proportion who agree that smoking can cause..." by smokingrelated diseases.

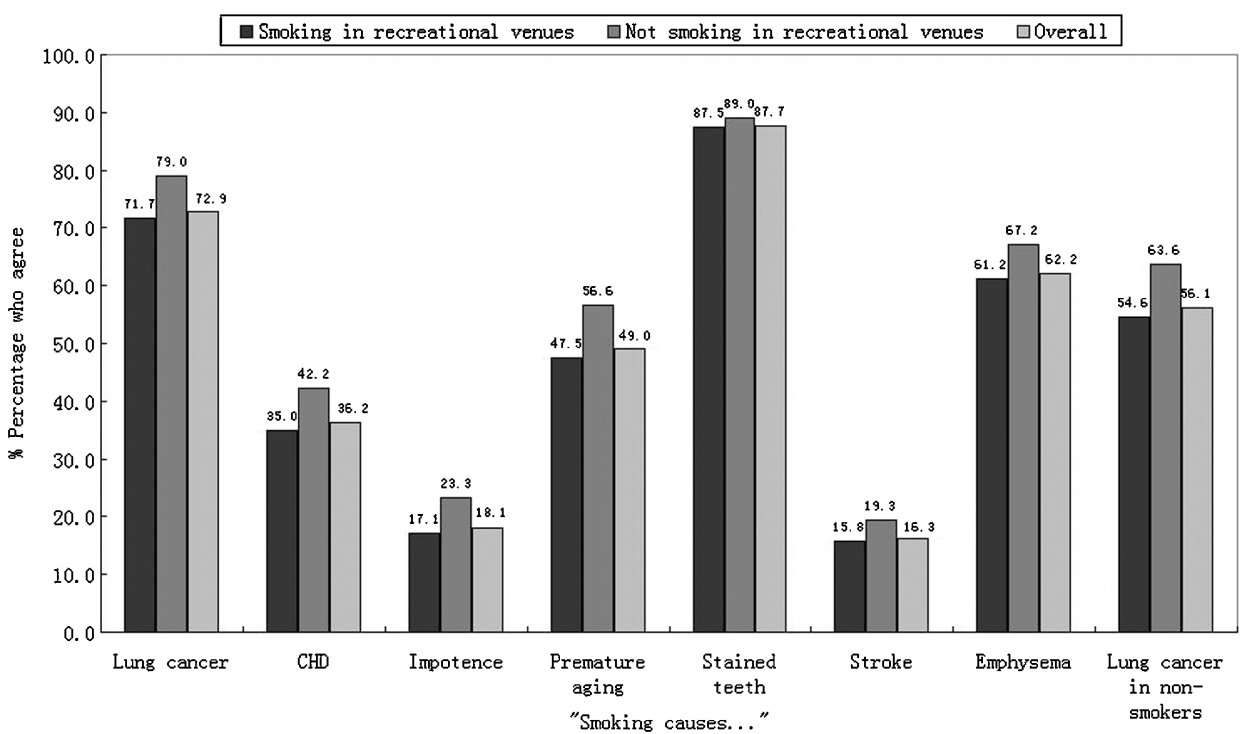


Table 3 Crude and adjusted odds ratio of recreational venues smoking risk factors and its $95 \%$ confidence interval

\begin{tabular}{|c|c|c|}
\hline Variables & Crude OR (95\% CI) & AOR $(95 \% \mathrm{Cl})^{*}$ \\
\hline \multicolumn{3}{|l|}{ Reported level of bans } \\
\hline Total indoor area & Reference & Reference \\
\hline Some indoor area & $3.12(2.21$ to 4.39$)$ & $3.14(2.18$ to 4.53$)$ \\
\hline No restrictions & 14.05 (9.28 to 21.28$)$ & 14.36 (9.41 to 21.91$)$ \\
\hline \multicolumn{3}{|l|}{ Support for indoor restrictions } \\
\hline Total indoor area & Reference & Reference \\
\hline Some indoor area & $1.31(1.00$ to 1.73$)$ & $1.34(1.01$ to 1.77$)$ \\
\hline DK & $1.61(0.92$ to 2.83$)$ & $1.62(0.93$ to 2.81$)$ \\
\hline No restrictions & $2.84(2.09$ to 3.86$)$ & 2.87 (2.12 to 3.89$)$ \\
\hline Believe smoking causes lung cancer in smokers & $0.63(0.47$ to 0.84$)$ & $0.62(0.45$ to 0.84$)$ \\
\hline Believe smoking causes CHD & $0.75(0.58$ to 0.95$)$ & $0.74(0.58$ to 0.96$)$ \\
\hline Believe smoking causes stroke & $0.86(0.63$ to 1.16$)$ & $0.86(0.63$ to 1.19$)$ \\
\hline Believe smoking causes impotence & $0.66(0.48$ to 0.91$)$ & $0.66(0.48$ to 0.91$)$ \\
\hline Believe smoking causes premature ageing & $0.69(0.54$ to 0.88$)$ & $0.68(0.53$ to 0.88$)$ \\
\hline Believe smoking causes stained teeth & $0.85(0.60$ to 1.22$)$ & $0.85(0.59$ to 1.21$)$ \\
\hline Believe smoking causes emphysema & $0.73(0.58$ to 0.92$)$ & $0.72(0.58$ to 0.90$)$ \\
\hline Believe smoking causes lung cancer in non-smokers & $0.68(0.51$ to 0.90$)$ & $0.68(0.51$ to 0.91$)$ \\
\hline \multicolumn{3}{|l|}{ Total number of health effects reported } \\
\hline$\leqslant 1$ & Reference & Reference \\
\hline 2 & $0.97(0.56$ to 1.67$)$ & $0.96(0.56$ to 1.66$)$ \\
\hline 3 & $0.86(0.55$ to 1.34$)$ & $0.84(0.55$ to 1.29$)$ \\
\hline 4 & $0.60(0.36$ to 0.10$)$ & $0.58(0.36$ to 0.95$)$ \\
\hline 5 & $0.61(0.43$ to 0.86$)$ & $0.60(0.41$ to 0.86$)$ \\
\hline 6 & $0.51(0.32$ to 0.81$)$ & $0.50(0.31$ to 0.79$)$ \\
\hline 7 & $0.57(0.36$ to 0.91$)$ & $0.56(0.34$ to 0.91$)$ \\
\hline 8 & $0.47(0.27$ to 0.82$)$ & $0.45(0.25$ to 0.81$)$ \\
\hline Trend test & $\chi_{\text {trend }}^{2}=28.4$ & $\mathrm{p}_{\text {trend }}<0.001$ \\
\hline \multicolumn{3}{|l|}{ Tobacco is addictive } \\
\hline Agree & Reference & Reference \\
\hline Neither disagree nor agree & $0.84(0.53$ to 1.35$)$ & $0.82(0.51$ to 1.30$)$ \\
\hline Disagree & $0.98(0.64$ to 1.48$)$ & $0.99(0.65$ to 1.51$)$ \\
\hline \multicolumn{3}{|l|}{ Enjoying smoking too much to give it up } \\
\hline Disagree & Reference & Reference \\
\hline Neither disagree nor agree & $1.01(0.61$ to 1.66$)$ & $1.13(0.74$ to 1.74$)$ \\
\hline Agree & $0.89(0.63$ to 1.25$)$ & $1.10(0.78$ to 1.55$)$ \\
\hline \multicolumn{3}{|l|}{ Overall opinion of smoking behaviour } \\
\hline Very good & 30.40 (3.93 to 235.46$)$ & 30.64 (3.94 to 238.25$)$ \\
\hline Good & 2.19 (1.17 to 4.07$)$ & 2.20 (1.16 to 4.18$)$ \\
\hline Neither good nor bad & $1.76(1.21$ to 2.56$)$ & $1.80(1.23$ to 2.63$)$ \\
\hline Bad & $1.20(0.86$ to 1.66$)$ & $1.24(0.90$ to 1.70$)$ \\
\hline Very bad & Reference & Reference \\
\hline \multicolumn{3}{|l|}{ Attitude of Chinese society to smoking } \\
\hline Disapprove/neither/DK & Reference & Reference \\
\hline Support & $1.27(1.00$ to 1.60$)$ & $1.27(1.00$ to 1.60$)$ \\
\hline \multicolumn{3}{|l|}{ Cigarettes per day } \\
\hline $0-10$ & Reference & Reference \\
\hline $11-20$ & 2.75 (2.15 to 3.52$)$ & 2.81 (2.17 to 3.64$)$ \\
\hline $21-30$ & $2.95(1.65$ to 5.26$)$ & 2.99 (1.67 to 5.33$)$ \\
\hline $31+$ & $2.30(1.40$ to 3.79$)$ & $2.43(1.48$ to 3.40$)$ \\
\hline \multicolumn{3}{|l|}{ Smokers of five closest friends } \\
\hline None & Reference & Reference \\
\hline One & $1.99(0.66$ to 6.01$)$ & $2.13(0.72$ to 6.32$)$ \\
\hline Two & $1.52(0.64$ to 3.64$)$ & $1.55(0.66$ to 3.61$)$ \\
\hline Three & $2.60(1.05$ to 6.41$)$ & $2.56(1.09$ to 6.05$)$ \\
\hline Four & 2.89 (1.07 to 7.83$)$ & 2.79 (1.08 to 7.23$)$ \\
\hline All & $4.05(1.70$ to 9.65$)$ & 3.81 (1.67 to 8.68$)$ \\
\hline \multicolumn{3}{|l|}{ Region } \\
\hline Beijing & Reference & Reference \\
\hline Shenyang & 2.80 (1.83 to 4.28$)$ & $2.74(1.75$ to 4.29$)$ \\
\hline Shanghai & 2.57 (1.83 to 3.63$)$ & $2.68(1.90$ to 3.79$)$ \\
\hline Changsha & 2.04 (1.46 to 2.84$)$ & 2.06 (1.45 to 2.92$)$ \\
\hline Guangzhou & $1.92(1.41$ to 2.60$)$ & $2.12(1.54$ to 2.92$)$ \\
\hline
\end{tabular}


Table 3 Continued

\begin{tabular}{lll}
\hline Variables & Crude OR (95\% Cl) & AOR (95\% CI)* \\
\hline $\begin{array}{l}\text { Yinchuan } \\
\text { Gender }\end{array}$ & $1.84(1.27$ to 2.67$)$ & $1.77(1.21$ to 2.61$)$ \\
$\begin{array}{l}\text { Male } \\
\text { Female }\end{array}$ & $\begin{array}{l}1.85(0.97 \text { to } 3.53) \\
\text { Reference }\end{array}$ & $\begin{array}{l}1.84(0.96 \text { to } 3.49) \\
\text { Reference }\end{array}$ \\
$\begin{array}{l}\text { Age } \\
18-24\end{array}$ & $2.74(0.97$ to 7.74$)$ & $3.27(1.05$ to 10.17$)$ \\
$25-39$ & $1.15(0.78$ to 1.70$)$ & $1.21(0.82$ to 1.80$)$ \\
$40-54$ & $1.74(1.11$ to 2.72$)$ & $1.74(1.10$ to 2.76$)$ \\
$55+$ & Reference & Reference \\
\hline
\end{tabular}

OR, odds ratio; $\mathrm{Cl}$, confidence interval; $\mathrm{AOR}$, adjusted odds ratio.

${ }^{*}$ Adjusted odds ratio: adjusting the potential confounding demographic characteristics including marital status, education attainment, ethnicity, and average monthly household (HH) income. DK, don't know.

particularly those living in middle-income and lower-income areas where smoke-free restrictions are non-existent.

\section{Implications}

Compared with Western countries, little has been done with regard to tobacco control in China, especially in recreational venues. Although only a first step, the findings from this survey present a valuable basis to move forward on tobacco control within recreational settings, by exploring the determinants of public smoking behaviour in these venues. All such information is of great importance in policy-making, which is urgently needed to decrease the high smoking prevalence within recreational settings. Results of this study call for a prevention policy aimed at this special population, and strategies to reduce smoking in recreational venues, as in smoking behaviour in general, should involve educating the Chinese public about the hazards of secondhand smoke, associating smoking behaviour with negative rather than positive images, and in trying to denormalise smoking. These are the strategies that have proved to be effective in many other countries, and provide a superb set of strategies for China to apply as it increases its efforts to combat the single most important cause of death and disability in the world's most populous country.

Acknowledgements: The authors would like to acknowledge the Chinese Center for Disease Control and Prevention and the local CDC representatives in each city for their role in data collection.

Funding: The ITC China Project was supported by grants from the US National Cancer Institute (R01 CA125116 and the Roswell Park Transdisciplinary Tobacco Use Research Center (P50 CA111236)), Canadian Institutes of Health Research (79551), Chinese Center for Disease Control and Prevention, and the Ontario Institute for Cancer Research.

Competing interests: None.

Provenance and peer review: Not commissioned; externally peer reviewed.

\section{REFERENCES}

1. Gajalaksmi C, Jha P, Ranson K, et al. Global patterns of smoking and smokingattributable mortality. In: Jha P, Chaloupka F, eds. Tobacco control in developing countries. New York: Oxford University Press, 2000:11-39.

2. Hammond D, Kin F, Prohmmo A, et al. Patterns of smoking among adolescents in Malaysia and Thailand: findings from the International Tobacco Control Southeast Asia Survey. Asia Pac J Public Health 2008;20:193-203.

3. Abdullah A, Husten C. Promotion of smoking cessation in developing countries: a framework for urgent public health interventions. Thorax 2004;59:623-30.

4. World Health Organization. WHO report on the global tobacco epidemic, 2008: the MPOWER package. Geneva: WHO, 2008.

5. Yang G. The epidemiologic investigation of the smoking behavior among Chinese population in 2002. Chinese Smoking and Health 2004;62:7-18.

6. Yang G, Ma J, Chen A, et al. Smoking cessation in China: findings from the 1996 national prevalence survey. Tob Control 2001:10:170-4.

7. China Tobacco Control and FCTC Fulfillment Summit. Report on tobacco control in China for 2008. Beijing, 2008 .
8. Doll R, Peto R, Boreham J, et al. Mortality in relation to smoking: 50 years observations on male British doctors. BMJ 2004;328:1519-27.

9. US Center for Disease Control and Prevention. School-based tobacco-use prevention-People's Republic of China, May 1989-Jan 1990. Morb Mort Wkly Rep 1993;42:377

10. China Tobacco Control and FCTC Fulfillment Summit. Report on tobacco control in China for 2007. Beijing, 2007.

11. Pierce JP, Choi WS, Gilpin EA, et al. Validation of susceptibility as a predictor of which adolescents take up smoking in the United States. Health Psychol 1996:15:355-61.

12. Choi WS, Pierce JP, Gilpin EA, et al. Which adolescent experimenters progress to established smoking in the United States. Am J Prev Med 1997;13:385-91.

13. Katz SK, Lavack AM. Tobacco-related bar promotions: insights from tobacco industry documents. Tob Control 2002;11(suppl I):i92-101.

14. Sepe E, Ling P, Glantz S. Smooth moves: bar and nightclub tobacco promotions that target young adults. Am J Public Health 2002;92:414-9.

15. Sepe E, Glantz SA. Tobacco promotions in alternative press: targeting young adults. Am J Public Health 2002;92:75-8.

16. Peto R, Chen Z, Boreham J. Tobacco- - the growing epidemic in China. JAMA 1996:275:1683-14.

17. Charlton A, Minagawa K, While D. Saying 'no' to cigarettes: a reappraisal of adolescent refusal skills. J Adolesc 1999;22:695-707.

18. CDC. Youth tobacco surveillance-United States, 1998-1999. CDC Surveillance Summaries 2000;49:1-94.

19. Epstein J, Griffin K, Botvin G. A model of smoking among inner-city adolescents: the role of personal competence and perceived social benefits of smoking. Prev Med 2000;31:107-14.

20. Wilkinson AV, Schabath MB, Arokhorov AV, et al. Age-related differences in factors associated with smoking initiation. Cancer Causes Control 2007;18:635-44.

21. Mermelstein R. Addicted to nicotine: ethnicity, gender, and risk factors for smoking initiation. Bethesda, MD: National Research Forum, 1998.

22. Wu C, Thompson ME, Fong GT, et al. Methods of the International Tobacco Control (ITC) China Survey. Tob Control 2010; 19(Suppl 2):i1-i5.

23. Binson D, Canchola JA, Catania JA. Random selection in a national telephone survey: a comparison of the Kish, next-birthday, and last-birthday methods. J Off Stat 2000; 16:53-60

24. Fong GT, Cummings KM, Borland $\mathrm{R}$, et al. The conceptual framework of the International Tobacco Control (ITC) Policy Evaluation Project. Tob Control 2006;15(suppl 3):iii3-11.

25. Thompson ME, Fong GT, Hammond D, et al. Methods of the International Tobacco Control (ITC) Four Country Survey. Tob Control 2006;15(suppl 3):iii12-8.

26. Borland R, Yong H, Siahpush M, et al. Support for and reported compliance with smoke-free restaurants and bars by smokers in four countries: findings from the International Tobacco Control (ITC) Four Country Survey. Tob Control 2006;15:34-41.

27. Clarke $\mathbf{J}$, Borland $\mathrm{R}, \mathrm{McG}$ artland $\mathrm{M}$. The effects of smoking outside workplaces on non-regular smokers. J Occup Environ Med 1997;39:734-9.

28. Brownson R, Hopkins D, Wakefield M. Effects of smoking restrictions in the workplace. Annu Rev Public Health 2002;23:333-48.

29. Parry 0, Platt S, Thomson C. Out of sight, out of mind: workplace smoking bans and the relocation of smoking at work. Health Promotion Int 2000;15:125-33.

30. Shopland D, Gerlach K, Burns D, et al. State-specific trends in smoke-free workplace policy coverage: the current population survey tobacco use supplement, 1993 to 1999. J Occup Environ Med 2001;43:680-6

31. Wakefield M, Roberts L, Owen N. Trends in prevalence and acceptance of workplace smoking bans among indoor workers in South Australia. Tob Control 1996;5:205-8.

32. Poland B, Stockton L, Ashley M, et al. Interactions between smokers and nonsmokers in public places: a qualitative study. Can J Public Health 1999;90:330-3.

33. Unger J, Rohrbach L, Howard K, et al. Attitudes toward anti-tobacco policy among California youth: associations with smoking status, psychosocial variables and advocacy actions. Health Educ Res 1999;14:751-63.

34. Ross N, Taylor S. Geographical variation in attitudes towards smoking: findings from the COMMIT communities. Soc Sci Med 1998:46:703-17. 
35. Borland R, Owen N, Hocking B. Changes in smoking behavior after a total workplace smoking ban. Aust J Public Health 1991;15:130-4.

36. Borland R, Owen N, Hill D, et al. Changes in acceptance of workplace smoking bans following their implementation: a prospective study. Prev Med 1990:19:314-22.

37. Janz $\mathbf{N}$, Becker MH. The health belief model: a decade later. Health Educ 0 1984;11:1-47.

38. Bandura A. Self-efficacy: toward a unifying theory of behavioral change. Psychol Rev 1977:84:191-215.

39. Ajzen I. The theory of planned behavior. Organ Behav Hum Decis Process 1991:50:179-211.

40. Smith M, Umenai T. Knowledge, attitude and practice of smoking among university students of allied health sciences in Japan. Asia Pac J Public Health 2000:12:17-21.

41. Rosliza A, Khadijah S. Smoking among young urban Malaysian women and its risk factors. Asia Pac J Public Health 2008;20:204-13.
42. Oncken C, McKee S, Sarin S, et al. Knowledge and perceived risk of related conditions: a survey of cigarette smokers. Prev Med 2004;10:10-6.

43. Aloise $\mathbf{Y}$, Hennigan K. Self-image, the smoker stereotype and cigarette smoking: developmental patterns from fifth through eighth grade. J Adolesc 1996:19:163-77.

44. Wang 0, Fitzhugh E, Eddy J, et al. Attitudes and beliefs of adolescent experimental smokers: a smoking prevention perspective. J Alcohol Drug Educ 1996;41:1-12.

45. Hill A, Bourdreau $\mathrm{R}$, Amyot $\mathrm{E}$, et al. Predicting the stages of smoking acquisition according to the theory of planned behavior. J Adolesc Health 1997;21:107-15.

46. Nevbahar Ertas. Factors associated with stages of cigarette smoking among Turkish youth. Eur J Public Health 2006:17:155-61.

47. Tyas S, Pederson L. Psychosocial factors related to adolescent smoking: a critical review of the literature. Tob Control 1998;7:409-20.

48. Bauman K, Ennett S. On the importance of peer influence for adolescent drug use: commonly neglected considerations. Addiction 1996;91:185-98. 